\title{
Clinical Cardiac Markers for Acute Myocardial Infarction Diagnostic
}

\author{
Winne Fernanda Souto Maior da Silva ${ }^{1}$, Vitória Regina Rodrigues de Oliveira ${ }^{1}$, Bárbara Virgínia \\ Mendonça da Silva Correia ${ }^{1 *}$ and Auvani Antunes da Silva Junior ${ }^{1,2}$ \\ ${ }^{1}$ Faculdade UniNassau, Brazil \\ ${ }^{2}$ Universidade Federal de Pernambuco, Brazil
}

*Corresponding author: Bárbara Virgínia Mendonça da Silva Correia, Biomedicine Department, BR 104, KM 68, n 1215 - Agamenon Magalhães, Caruaru - PE, 55000-000, Brazil.

To Cite This Article: Winne Fernanda Souto Maior da Silva, Vitória Regina Rodrigues de Oliveira, Bárbara Virgínia Mendonça da Silva Correia, Auvani Antunes da Silva Junior. Clinical Cardiac Markers for Acute Myocardial Infarction Diagnostic. Am J Biomed Sci \& Res. 2021 - 13(5). AJBSR. MS.ID.001920. DOI: 10.34297/AJBSR.2021.13.001920.

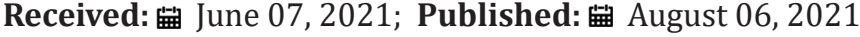

\begin{abstract}
Cardic biomarkers are biological macromolecules that can be used in laboratory routine and clinical as confirmatory evidence for diagnostic of the Acute Myocardial Infarction (AMI). Several cardiac biomarkers, such as cardiac enzymes and especific cardio proteins, have been tradiocionally used in the AMI diagnostic. However, the difference between the sensitivity and specificity of the biomarker of clinical choice can be assistance of in the early diagnosis and the evaluation prognosis of the infarcted patient. The purpose of this review is to summarize an update of the clinical cardic biomarker used in the diagnostic of AMI, specifying the criterion of sensitivity, tissue performance and its correlation regarding the diagnosis.
\end{abstract}

Keywords: Cardiovascular Diseases, Acute Myocardial Infarction, Cardiac Biomarkers, Diagnosis

\section{Introduction}

Cardiovascular Diseases (CVDs) represent 31\% of the world mortality rate, according to the World Health Organization, with an estimated 17.9 million deaths per year [1]. CVDs are associated with numerous non-modifiable risk factors such as age, sex, race, and family genetics and modifiable, such as unbalanced diet, alcoholism, smoking, stress, and sedentary lifestyle [2]. The CVDs include coronary heart disease (such as acute coronary syndromes and heart failure), cerebrovascular disease, rheumatic heart disease and other conditions. Among the acute coronary syndromes, Acute Myocardial Infarction (AMI) is classified as one of the main causes of hospitalizations and deaths in the world [3].

AMI refers to acute focal myocardial necrosis caused by prolonged and severe ischemia, which can progress by reducing oxygen consumption in the myocardium [4]. The most deaths from AMI occur during the first $4 \mathrm{~h}$ of myocardial necrosis. If the patient is diagnosed and treated effectively within the first few $\mathrm{h}$, mortality can be reduced from $9 \%$ to $3 \%$, but if diagnosed within $3-4 \mathrm{~h}$, the mortality rate can be five times higher [5]. Therefore, agility in the diagnosis is essential for the therapeutic conduct and survival of the infarcted patient.

According with the Universal Definition of Myocardial Infarction [4], the diagnosis of the AMI requires the clinical history of the patient associated with clinical symptoms, electrocardiogram (ECG) tracing and cardiac markers. The ECG is the main diagnostic method used in the screening; it must be performed in less than 10 min from presentation to the emergency. However, a negative percentage of infarcted patients do not present changes in the ST segment of the ECG, reducing their sensitivity to $50-60 \%$ for AMI diagnostic [6,7]. A rapid and accurate diagnosis is essential for effective medical management and revascularization of the infarcted patients. In this sense, the use elevation in serum concentration of cardiac biomarkers is of paramount importance for stratifying risk and prognosis of the AMI. 
Cardiac markers are described as protein molecules of the cellular structure detectable in the bloodstream when damage to cardiac cells occurs. The release kinetics of the cardiac markers in AMI depends on several factors, such as size of molecules, clearance rate of the marker, analytical method used for its measurement and, above all, of its sensitivity. There are numerous cardiac biomarkers, it is useful to classify biomarkers into various pathophysiologic groups, such as myocardial ischemia or necrosis, inflammation, hemodynamics, angiogenesis, atherosclerosis, or plaque instability [8]. Traditionally, some cytosolic enzymes were measured as indicators of the cardiac ischemia and infarction. Currently, other constituent biochemical markers muscle cell proteins without enzyme function have also been used for this purpose.

The ideal biomarker for detecting myocardial injury needs to be expressed at relatively high levels within cardiac tissue, with high clinical sensitivity and specificity that is detectable in the blood early after the onset of symptoms, such as chest pain $[8,9]$. The choice of the cardiac biomarkers associated with point-ofcare testing technology demonstrate a potential strategy for the rapid and practical management and cost reduction of the AMI diagnostic. The current article shows an integrative review about of the sensibility and specificity of mainly cardiac biomarkers used for AMI diagnostic and its use of the in clinical practice. The main molecular markers used in clinical practice for the diagnosis of AMI are enzyme Creatine Kinase (CK), Myoglobin (Mb), Cardiac Fatty Acid Binding Protein (H-FABP), Brain Natriuretic Peptide (BNP) and Cardiac Troponins T and I (cTnT and cTnI).

\section{Creatine Kinase in the AMI Diagnostic}

$\mathrm{CK}$ is a cytoplasmic protein that catalyzes the reversible phosphorylation of creatine to phosphocreatine, generating anaerobic muscle contraction, common in ischemic events. It consists of two subunits ( $\mathrm{M}$ and $\mathrm{B}$ ), forming three isomers (CK-BB, CK-MB and CK-MM). The CK-MM isoenzyme is more abundant in the heart and skeletal muscle tissue; CK-BB derived from the brain, lungs, and smooth tissues; CK-MB originating predominantly in the myocardium [10]. Serum CK-MB levels rise 3-6h after precordial chest pain, reaching a peak in $24 \mathrm{~h}$ and normalizing within $48-72 \mathrm{~h}$. The CK-MB has a sensitivity of $50 \%$ after $3 \mathrm{~h}$ of onset of symptoms and $80 \%$ after $6 \mathrm{~h}$ [11]. Therefore, its increasing level during trauma and inflammation reduces its specificity due to the falsepositive correlation with non-cardiac tissues [10,11]. Additionally, the levels of CK-MB concentrations can not detect minor damage in myocardial tissue, due to the high molecular weight.

\section{Myoglobin in the AMI Diagnostic}

$\mathrm{Mb}$ is a cytoplasmic hemoprotein of low molecular weight (17 $\mathrm{kDa}$ ) with a structure like that of hemoglobin. $\mathrm{Mb}$ is the earliest cardiac marker that changes and normalizes in the bloodstream after an ischemic injury. The serum MB levels rise rapidly after myocardial injury, their elevation occurs between 30min after the onset of ischemia, reaching its maximum around 6-10h and normalizing between 12-24h $[12,13]$. The Mb can be used for evaluation of the infarct size and reperfusion [14]. Mb has low specificity for cardiac tissue, especially in patients with renal failure and trauma to the skeletal muscles, chronic insufficiency, exposure to drugs and toxins. However, it should be noted that it is useful in evaluating infarct size and reperfusion.

\section{Cardiac Troponins in the AMI Diagnostic}

Currently, cardiac troponins are the "gold standard" markers for diagnosis of ischemic myocardial lesions. Due to its high specificity and sensitivity became a consensus since the first redefinition of the AMI proposed by a committee by the European Society of Cardiology and the American College of Cardiology [4]. Recent revisions of the committee considered the importance of serial dosing of troponins in the AMI, reiterating its position as a differential and first-choice marker. The cTnT and cTnI are expressed as isoforms specific to cardiac muscle. These become measurable within a period of 2 to $4 \mathrm{~h}$ after the onset of symptoms clinical and remains elevated for about 4 to 7 days for cTnI, and 10 to 14 days for cTnT $[15,16]$. Some authors attribute to cTnI and cTnT the same specificity and importance in the evaluation of cardiac damage [17]. However, the cTnT has a wider diagnostic window and compared to cTnI, allowing the assessment of both the acute and chronic phases of AMI.

\section{Other Cardiac Proteins in the AMI Diagnostic}

Recently, others cardiac biomarkers have been described in the literature with the aim of improving the diagnostic specificity of the infarction and the prognostic of the myocardial damage. Among them, the H-FABP and BNP serum concentrations and have shown potential application to improve the accuracy and sensitivity of infarction diagnosis in emergency departments.

The H-FABP protein is released from $20 \mathrm{~min}$ after the beginning of the necrosis process and reaches its peak concentration after 4-6h. This allows the use of H-FABP as a cardiac marker within the first hours of the infarction [18]. In this sense, it is important to be evaluated in association with other markers, improving the accuracy of the diagnosis. The H-FABP provides increase in the sensitivity and negative predictive value for patients with chest pain and no cTn elevations, showing a higher sensitivity in the diagnosis of AMI with a positive rate of 55\% [19].

However, the high sensibility of the cTn blood levels in the AMI diagnostic, its concentration on admission of the patients was weakly associated with the infarct size estimation [20]. The BNP blood concentration can be used as an important predictor for left 
ventricular dysfunction after the onset of AMI and a good indicator of infarct size and monitoring of the damage tissue. The BNP is a cardiac neurohormone released from ventricles in response to myocardial dysfunction [21]. In this sense, the BNP serum concentration associated with cTn showed a good correlation for immediate infarct severity estimation and risk stratification of the ventricular dysfunction [22].

\section{Conclusion}

The rapid diagnosis of infarction is crucial for clinical decision the appropriate therapeutic treatment that can be improve the patient prognostic. Serum measurement of cardiac biomarkers helps clinical practice, especially when other decision-making events such as characteristic symptoms and ECG changes are inconclusive. Although there are many cardiac biomarkers, their use in clinical practice depends on their specificity/sensitivity to detect myocardial damage, reproducibility, precision, and discriminatory limits to distinguish between pathological and physiological levels.

\section{Conflict of Interest}

The authors declare no conflict of interest.

\section{References}

1. WHO (2020) Cardiovascular diseases.

2. Mensah GA, Roth GA, Fuster V, Johnson CO, Addolorato G, et al. (2020) The global burden of cardiovascular diseases and risk factors. Journal of the American College of Cardiology 76(25): 2982-3021.

3. Reynolds K, Go AS, Leong TK, Boudreau DM, Cassidy Bushrow AE, et al. (2017) Trends in incidence of hospitalized acute myocardial infarction in the Cardiovascular Research Network (CVRN). The American journal of medicine 130(3): 317-327.

4. Thygesen K, Alpertet JS, Jaffeal AS, Chaitman BR, Bax JJ, et al. (2018) Fourth Universal Definition of Myocardial Infarction (2018) Circulation. Circulation 138(20): 618-651.

5. Alabas OA, Jernberg T, Pujades Rodriguez M, Rutherford MJ, West RM, et al. (2020) Statistics on mortality following acute myocardial infarction in 842897 Europeans. Cardiovascular research 116(1): 149-157.

6. Montecucco F, Carbone F, Schindler TH (2016) Pathophysiology of ST-segment elevation myocardial infarction: novel mechanisms and treatments. European heart journal 37(16): 1268-1283.

7. Roffi M, Patrono C, Collet JP, Mueller C, Valgimigli M, et al. (2015) ESC guidelines for the management of acute coronary syndromes in patients presenting without persistent ST-segment elevation. Eur Heart J V 37(3): 267-315.

8. Xi Ying W, Zhang F, Zhang C, Zheng LR, Yang J, et al. (2020) The biomarkers for acute myocardial infarction and heart failure. BioMed research international pp:14.
9. Tilea I, Varga A, Serban RC (2021) Past, Present, and Future of Blood Biomarkers for the Diagnosis of Acute Myocardial Infarction-Promises and Challenges. Diagnostics 11(5): 881.

10. Wiens E, Arbour J, Seifer C (2018) Measurement of Creatine Kinase in the Emergency Department for Diagnosis of Acute Myocardial Infarction. Canadian Journal of Cardiology 34(10): 74.

11. Man C, et al. (2017) Meta-analysis of remote ischemic conditioning in patients with acute myocardial infarction. Scientific reports 7(1): 1-9.

12. Kottwitz, J, Berg J, Elhassan M, Baltensperger N, Kissel CK, et al. (2020) Myoglobin for detection of high-risk patients with acute myocarditis. Journal of cardiovascular translational research 138(5): 853-863.

13. Adidharma IF, Nugraha J, Aminuddin M (2020) The Association Between Myoglobin, Troponin I, Hfabp and Nt-Probnp Levels with Acute Myocardial Infarction in Patients with Acute Coronary Syndrome. Indian Journal of Forensic Medicine \& Toxicology 14(2): 2020.

14. Aydin S, Ugur K, Aydin S, Sahin I, Yardim M (2019) Biomarkers in acute myocardial infarction: current perspectives. Vascular health and risk management 15: 1-10.

15. Kvisvik B, Mørkrid L, Røsjø H, Cvancarova M, Rowe AD, et al. (2017) Highsensitivity troponin T vs I in acute coronary syndrome: prediction of significant coronary lesions and long-term prognosis. Clinical Chemistry 63(2): 552-562.

16. Procopio A, Rosa SD, Covello C, Merola A, Sabatino J, et al. (2017) A model of cardiac troponin $\mathrm{T}$ release in patient with acute myocardial infarction. 2017 IEEE 56th Annual Conference on Decision and Control (CDC), Melbourne VIC p:435-440.

17. Welsh P, Preiss D, Hayward C, Shah ASV, McAllister D, et al. (2018) Comparison between high-sensitivity cardiac troponin $\mathrm{T}$ and cardiac troponin I in a large general population cohort. Clinical chemistry 64(11): 1607-1616.

18. Agnello, L. et al. (2017) Heart-type fatty acid binding protein is a sensitive biomarker for early AMI detection in troponin negative patients: a pilot study. Scandinavian journal of clinical and laboratory investigation $77(6): 428-432$

19. Dupuy AM, Cristol JP, Kuster N, Reynier R, Lefebvre S, et al. (2015) Performances of the heart fatty acid protein assay for the rapid diagnosis of acute myocardial infarction in ED patients. The American Journal of Emergency Medicine 33(3): 326-330.

20. Cummins B, Auckland ML, Cummins P (1987) Cardiac-specific troponin-l radioimmunoassay in the diagnosis of acute myocardial infarction. American heart journal 113(6): 1333-1344.

21. Shen F, Chengfang Z, Juan Z, Guangxin C, Hui L, et al. (2017) Clinical study of combined detection of troponin T, myoglobin and NT-ProBNP in diagnosis of myocardia linfarction. International Journal of Laboratory Medicine 38(9): 1220-1221.

22. Israr MZ, Liam MH, Leong LN, Toru S (2018) B-type natriuretic peptide molecular forms for risk stratification and prediction of outcome after acute myocardial infarction. American heart journal 200: 37-43. 